\title{
ON THE REDUCTION OF INTEGRO-DIFFERENTIAL EQUATIONS*
}

\author{
BY \\ GRIFFITH C. EVANS
}

Certain integro-differential equat ons can be completely treated by first resolving an integral equation, and second, integrating a differential equation. In so far, such equations do not constitute a new problem in analysis, because they are reducible to equations of simpler types. From this point of view Volterra discusses the equation

$$
\nabla^{2} V(x, y, z \mid t)+\int_{t_{0}}^{t} f(t, \tau) \nabla^{2} V(x, y, z \mid \tau) d \tau=F(x, y, z, t)
$$

which is satisfied by the potential $V$ of an electric field in an isotropic medium, in the so-called "static case" of hysteresis. $\dagger$ By solving this equation, regarded as an integral equation, for $\nabla^{2} V$, we are led to the differential equation of Poisson to determine $V$.

We may however have the case that an integro-differential equation whose solution is subject to certain boundary conditions is reducible to one or more problems in equations of more elementary character only when account is taken of these conditions. This is the point of view to be developed in the present paper. It is a point of view which can be extended to more general types of functional equations.

We shall treat, in the following pages, the reduction of boundary value problems in the case of the integro-differential equation of parabolic type, and quite briefly that of hyperbolic type, to problems in linear differential and integral equations separately. It is worth while perhaps to specially mention $\S 2$, in which the generalization of the integro-differential equation of the second order to an equation of the first order involving integration

\footnotetext{
* Presented to the Society, December, 1912, and December, 1913. Since the first writing, the paper has been entirely revised, partly to include new material, and partly to take advantage of the material of Prof. W. A. Hurwitz's thesis, Randwertaufgaben bei Systemen von linearen partiellen Differentialgleichungen erster Ordnung, D is s. G ö t t ing e n, 1910, of which the author was ignorant at the time of the first writing.

$\dagger \mathrm{V}$. Volterra, Lectures delivered at the celebration of the 20th anniversary of the foundation of Clark University (Sept., 1911), p. 75; Sulle equazioni integro differenziali, R e n d i c o n t i d ella R. A c c a de mia de i L in c e i, vol. 18 (1909), p. 174; Sulle equazioni integrodifferenziali della teoria dell'elasticità, ibid., vol. 18 (1909), p. 297.
} 
around an arbitrary path demands a corresponding restatement and treatment of the non-homogeneous linear partial differential equation of parabolic type.*

\section{The Integro-Differential Equation of Parabolic Type}

1. The region and its corresponding equation. For the parabolic differential equation the earliest boundary value problems referred to an open rectangle as the boundary. Afterwards the results were extended to curves of a more general nature.

In connection with the integro-differential equation

$$
\frac{\partial u(x, t)}{\partial t}-\frac{\partial^{2} u(x, t)}{\partial x^{2}}=\int_{t_{0}}^{t} A(t, \tau) \frac{\partial u(x, \tau)}{\partial \tau} d \tau
$$

there is a boundary value problem based upon the open rectangle: if under proper conditions a chain of values is given along the three sides of this rectangle, the given equation then uniquely determines them along the fourth side. In fact, for all the partial integro-differential equations heretofore considered, the boundaries have been of this sort. And if we wish to extend the results to a more general type of region, it is also opportune for us, as it turns out, to consider a slightly more general type of integro-differential equation.

We shall take as the open boundary the curves $t=t_{0}$ on the left, and the curves $x=\xi_{2}(t)$ above, and $x=\xi_{1}(t)$ below. We shall assume that $\xi_{1}(t)$ and $\xi_{2}(t)$ and their first and second derivatives remain single valued, finite and continuous; moreover that $\xi_{2}(t)>\xi_{1}(t)$ for any value of $t \geqq t_{0}$. The derivatives $\xi_{1}^{\prime}(t)$ and $\xi_{2}^{\prime}(t)$ are to vanish only a finite number of times in the interval $t_{0} \leqq t \leqq t_{1}$. For simplicity we shall impose also the unnecessary restriction that $x=\xi_{1}(t)$ shall have no maximum and $x=\xi_{2}(t)$ no minimum, in the interval $t_{0}<t<t_{1} . \dagger$

We shall adopt Professor Hurwitz's convenient notation ${ }_{a} \Omega_{b}$ for the space bounded by these curves and by the straight lines $t=a$ on the left and $t=b$ on the right.

Along the open boundary is to be given the continuous chain of values

$$
\text { ( } \alpha) \quad F\left(x, T_{x}\right)=\left\{\begin{array}{ll}
f(x) & \text { when } t=t_{0} \\
\phi_{1}(t) & \text { when } x=\xi_{1}(t) \\
\phi_{2}(t) & \text { when } x=\xi_{2}(t)
\end{array}\right\} \quad\left[\xi_{1}\left(t_{0}\right) \leqq x \leqq \xi_{2}\left(t_{0}\right)\right]
$$

* For an extensive treatment of the differential equation of parabolic type see Hurwitz, loc. cit., on which our $\S 1$ is based, and also for a treatment that demands different conditions E. E. Levi, Sul l'equazione del calore, A n n a li d i M a t e m a t i c a, vol. 14 (1908), p. 187. $\dagger$ This restriction may be removed by an extension of the present method of proof, or by a continuous prolongation of the equation and its solution. 
where the functions $f(x), \phi_{1}(t)$, and $\phi_{2}(t)$ are supposed to have continuous first derivatives in the range under consideration, and $T_{x}$ is used to denote the $t$-coördinate of the left-hand point on the boundary whose other coördinate is $x$. When it is necessary to use the $t$-coördinate of the right-hand boundary point whose other coördinate is $x$, we shall denote it by $t_{x}, t_{1 x}$, etc., according as the right-hand limit of the region is formed by $t=t, t=t_{1}$, etc.

In connection with this region and these boundary values, we shall investigate the equation

$$
\frac{\partial u(x, t)}{\partial t}-\frac{\partial^{2} u(x, t)}{\partial x^{2}}=\int_{T_{x}}^{t} A(x, t, \tau) \frac{\partial u(x, \tau)}{\partial \tau} d \tau .
$$

We shall say that a function $u(x, t)$ is regular with respect to this equation if within the region and on its boundary $u$ and $\partial u / \partial x$ remain finite and continuous, and within the region $\partial^{2} u / \partial x^{2}$ and $\partial u / \partial t$ exist except on a regularly distributed set of curves, ${ }^{*}$ and are integrable linearly in the Riemann sense.

2. The theorem of reducibility for equation (1). In equation (1) let $A(x, t, \tau)$ and its first partial derivatives with respect to the three arguments be finite and continuous functions within and on the boundary of the region, and inside that boundary let $\partial A(x, t, \tau) / \partial \tau=A_{1}(x, t, \tau)$ have a derivative in regard to $x$ that remains finite and continuous, except on a regularly distributed set of curves.

Under these conditions, there is one and only one regular solution $u(x, t)$ of the equation (1), which joins on continuously to the boundary values $(\alpha)$. It can be reduced to the form

$$
u\left(x_{1}, t_{1}\right)=u^{\prime}\left(x_{1}, t_{1}\right)-\iint_{t_{0} \Omega_{t_{1}}} k\left(x_{1}, t_{1} \mid x, t\right) u^{\prime}(x, t) d x d t,
$$

in which $u^{\prime}(x, t)$ is the regular solution of the differential equation

$$
\frac{\partial u^{\prime}(x, t)}{\partial t}-\frac{\partial^{2} u^{\prime}(x, t)}{\partial x^{2}}=-A\left(x, t, T_{x}\right) F\left(x, T_{x}\right)
$$

which takes on the boundary values $(\alpha)$, and $k\left(x_{1}, t_{1} \mid x, t\right)$ is the function associated to

$$
\begin{aligned}
& K\left(x_{1}, t_{1} \mid x, t\right)=\frac{1}{2 \sqrt{\pi}}\left\{g\left(x, t \mid x_{1}, t_{1}\right) A(x, t, t)\right. \\
&\left.-\int_{t}^{t_{1 x}} A_{1}(x, \tau, t) g\left(x, \tau \mid x_{1}, t_{1}\right) d \tau\right\}
\end{aligned}
$$

by means of Volterra's relation

* See M. Bôcher, Introduction to Integral Equations (1909), p. 3.

Trans. Amer. Math. Soc. 32 


$$
\begin{aligned}
k\left(x_{1}, t_{1} \mid x, t\right)+K\left(x_{1}, t_{1} \mid x, t\right) & =\iint_{\Omega_{t_{1}}} K\left(x_{1}, t_{1} \mid \xi, \tau\right) k(\xi, \tau \mid x, t) d \xi d \tau \\
& =\iint_{\Omega_{\Omega_{1}}} k\left(x_{1}, t_{1} \mid \xi, \tau\right) K(\xi, \tau \mid x, t) d \xi d \tau
\end{aligned}
$$

By $g\left(x, t \mid x_{1}, t_{1}\right)$ is denoted the Green's function for the equation

$$
\frac{\partial u}{\partial t}=\frac{\partial^{2} u}{\partial x^{2}} *
$$

It shou'd be noticed that $u^{\prime}(x, t)$ depends on the boundary conditions, but that $K\left(x_{1}, t_{1} \mid x, t\right)$ is independent of them, and is determined merely by the coefficients of the equation and the nature of the region. We shall now give a proof of this theorem.

3. The equivalence of the integral and the integro-differential equations. By means of an integration by parts, the equation (1) may be rewritten in the form

$$
\begin{aligned}
\frac{\partial u(x, t)}{\partial t}-\frac{\partial^{2} u(x, t)}{\partial x^{2}}=A(x, t, t) u(x, t) & -A\left(x, t, T_{x}\right) F\left(x, T_{x}\right) \\
& -\int_{T_{x}}^{t} A_{1}(x, t, \tau) u(x, \tau) d \tau .
\end{aligned}
$$

We have the theorem: $\dagger$ If $\phi(x, y)$ is continuous in the given region, and if in the neighborhood of a point $\left(x_{1}, y_{1}\right)$ within that region $\partial \phi / \partial x$ exists and is continuous, then the function

$$
\bar{u}\left(x_{1}, t_{1}\right)=\frac{1}{2 \sqrt{\pi}} \iint_{t_{0} \Omega_{t_{1}}} g\left(x, t \mid x_{1}, t_{1}\right) \phi(x, t) d x d
$$

is regular in the neighborhood of the point $\left(x_{1}, y_{1}\right)$ and in that neighborhood satisfies the differential equation

$$
\frac{\partial u}{\partial t}-\frac{\partial^{2} u}{\partial x^{2}}=\phi(x, t) .
$$

Moreover it vanishes on the open boundary of the region $\Omega$.

If we examine the function $A\left(x, t, T_{x}\right) F\left(x, T_{x}\right)$ we notice that its

* We may write

where $g^{\prime}$ is regular and

$$
g\left(x, t \mid x_{1}, t_{1}\right)=h_{01 / 2}+g^{\prime},
$$

$$
h_{a \beta}\left(x, t \mid x_{1}, t_{1}\right)=\frac{\left(x_{1}-x\right)^{a}}{\left(t_{1}-t\right)^{\beta}} e^{-\frac{\left(x_{1}-x\right)^{2}}{4\left(t_{1}-t\right)}}
$$

(see for instance loc. cit. Levi, \& 7).

$\dagger$ This is a slight but obvious generalization of Hurwitz's theorem VIII $b$ (Hurwitz, loc. cit., p. 85), to which the same proof applies. 
derivative in regard to $x$ exists and is continuous except on a finite number of lines parallel to the $t$-axis, namely, those lines which pass through points where $\xi_{1}^{\prime}(t)$ or $\xi_{2}^{\prime}(t)$ vanishes, and the two lines $x=\xi_{1}\left(t_{0}\right)$ and $x=\xi_{2}\left(t_{0}\right)$. Hence we know that the regular solution $u^{\prime}(x, t)$ of (3), which satisfies the given conditions $(\alpha)$, exists and is uniquely determined. If we write then $u(x, t)=u^{\prime}(x, t)+v(x, t)$ we have the following integro-differential equation to determine $v(x, t)$;

$$
\begin{array}{r}
\frac{\partial v(x, t)}{\partial t}-\frac{\partial^{2} v(x, t)}{\partial x^{2}}=A(x, t, t) v(x, t)-\int_{T_{x}}^{t} A_{1}(x, t, \tau) v(x, \tau) d \tau \\
+A(x, t, t) u^{\prime}(x, t)-\int_{T_{x}}^{t} A_{1}(x, t, \tau) u^{\prime}(x, \tau) d \tau
\end{array}
$$

with the condition that it be regular, and join on continuously to zero boundary values.

By means of the customary analysis with Green's theorem, we find as a necessary condition on $v(x, t)$ that it satisfy the equation

$$
\begin{aligned}
v\left(x_{1}, t_{1}\right)=\frac{1}{2 \sqrt{\pi}} \iint_{t_{0} \Omega_{t_{1}}} g & \left(x, t \mid x_{1}, t_{1}\right)\{A(x, t, t) v(x, t) \\
& \left.-\int_{T_{x}}^{t} A_{1}(x, t, \tau) v(x, \tau) d \tau\right\} d x d t \\
& +\frac{1}{2 \sqrt{\pi}} \iint_{t_{0} \Omega_{t_{1}}} g\left(x, t \mid x_{1}, t_{1}\right)\left\{A(x, t, t) u^{\prime}(x, t)\right. \\
& \left.-\int_{T_{x}}^{t} A_{1}(x, t, \tau) u^{\prime}(x, \tau) d \tau\right\} d x d t
\end{aligned}
$$

whence, if we add $u^{\prime}\left(x_{1}, t_{1}\right)$ to both members, it follows that we must have

$$
\begin{array}{r}
u\left(x_{1}, t_{1}\right)=u^{\prime}\left(x_{1}, t_{1}\right)+\frac{1}{2 \sqrt{\pi}} \iint_{t_{0} \Omega_{t_{1}}} g\left(x, t \mid x_{1}, t_{1}\right)\{A(x, t, t) u(x, t) \\
\left.-\int_{T_{x}}^{t} A_{1}(x, t, \tau) u(x, \tau) d \tau\right\} d x d t .
\end{array}
$$

This, by a permissible change in the order of integration, since $g\left(x, t \mid x_{1}, t_{1}\right)$ is a continuous function divided by $\sqrt{t_{1}-t}$, may be written in the form

$$
u\left(x_{1}, t_{1}\right)=u^{\prime}\left(x_{1}, t_{1}\right)+\iint_{t_{0} \Omega_{t_{1}}} K\left(x_{1}, t_{1} \mid x, t\right) u(x, t) d x d t,
$$

where $K\left(x_{1}, t_{1} \mid x, t\right)$ is given by (4). It is necessary then that $u\left(x_{1}, t_{1}\right)$ be a solution of (10) within the region. 
On the other hand if $u\left(x_{1}, t_{1}\right)$ is continuous and a solution of (10), it will be regular and a solution of (1), and will join on continuously to the boundary values $(\alpha)$. In fact

$$
\begin{aligned}
\frac{\partial u}{\partial x_{1}}=\frac{\partial u^{\prime}}{\partial x_{1}}+\frac{1}{2 \sqrt{\pi}} \iint_{t_{0} \Omega_{t_{1}}} \frac{\partial g\left(x, t \mid x_{1}, t_{1}\right)}{\partial x_{1}}\{ & A(x, t, t) u(x, t) \\
& \left.-\int_{T_{x}}^{t} A_{1}(x, t, \tau) u(x, \tau) d \tau\right\} d x d t,
\end{aligned}
$$

and is therefore finite and continuous. Hence if we put

$$
\phi(x, t)=A(x, t, t) u(x, t)-\int_{T_{x}}^{t} A_{1}(x, t, \tau) u(x, \tau) d \tau
$$

we see that

$$
\begin{aligned}
\frac{\partial \phi(x, t)}{\partial x}=\frac{\partial}{\partial x}[A(x, t, t) u(x, t)]-\int_{T_{x}}^{t} \frac{\partial}{\partial x}[ & \left.A_{1}(x, t, \tau) u(x, \tau)\right] d \tau \\
& +A_{1}\left(x, t, T_{x}\right) u\left(x, T_{x}\right) \frac{d T_{x}}{d x}
\end{aligned}
$$

represents a function of $x, t$ which is continuous except for discontinuities regularly distributed. On some of these lines of discontinuity $\partial \phi / \partial x$ will become infinite. But referring to the theorem of Professor Hurwitz already quoted, we see that the function $\bar{u}(x, t)$ determined by giving $\phi(x, t)$ the value from (11) will be regular and a solution of equation (7), which takes on zero boundary values. Moreover, as we see from equation (9) this $\bar{u}(x, t)$ is precisely $u(x, t)-u^{\prime}(x, t)$ where $u(x, t)$ is the supposed continuous solution of (10), and $u^{\prime}(x, t)$ is the $u^{\prime}(x, t)$ already specified. Hence $u(x, t)$ will satisfy (6), and therefore (1), will be regular, and will take on the given boundary values $(\alpha)$. Our assertion is therefore proved.

It may be noticed here that the discontinuities in the derivatives of highest order in the integro-differential equation (1) appear as projected through the region from points on the boundary, on account of the dependence of the equation on the chosen region, a fact which has no analog in the theory of the parabolic differential equation.

4. The integral equation (10) and its solution in closed form. It merely remains to show that (10) has a unique continuous solution, which can be written in the form (2).

We can show directly that equation (10) has one and only one continuous solution. In fact, the solution may be written in the form of an absolutely and uniformly convergent series of continuous functions*

* Evans, Sull'equazione integro-differenziale di tipo parabolico, R e n di c on ti della R. A c c a d e mia d e i Lin ce i, vol. 21 (1912), p. 28. The proof applies with obvious modifications to this case. 


$$
u\left(x_{1}, t_{1}\right)=\sum_{n=1}^{\infty} u^{(n)}\left(x_{1}, t_{1}\right),
$$

where

$$
\begin{aligned}
& u^{(n)}\left(x_{1}, t_{1}\right)=\iint_{t_{0} \Omega_{t_{1}}} K\left(x_{1}, t_{1} \mid x, t\right) u^{(n-1)}(x, t) d x d t, \quad n>1, \\
& u^{(1)}\left(x_{1}, t_{1}\right)=u^{\prime}\left(x_{1}, t_{1}\right) .
\end{aligned}
$$

In order to reduce the solution to the closed form (3), we go through a process which incidentally gives us another proof of its existence. We must determine the function $k\left(x_{1}, t_{1} \mid x, t\right)$ which satisfies Volterra's relation (equation (5)).

To this purpose let us consider the series formed in the usual way

$$
-\sum_{1}^{\infty} K_{i}\left(x_{1}, t_{1} \mid x, t\right)
$$

in which

$$
\begin{aligned}
& K_{1}\left(x_{1}, t_{1} \mid x, t\right)=K\left(x_{1}, t_{1} \mid x, t\right) \\
& K_{i}\left(x_{1}, t_{1} \mid x, t\right)=\iint_{\Omega_{t_{1}}} K\left(x_{1}, t_{1} \mid \xi, \tau\right) K_{i-1}(\xi, \tau \mid x, t) d \xi d \tau .
\end{aligned}
$$

Of this series every term but the first remains finite. In fact for the second term, since from the explicit expression for $K\left(x_{1}, t_{1} \mid x, t\right)$

in which $M$ is some constant, we have

$$
\left|K\left(x_{1}, t_{1} \mid \xi, \tau\right)\right| \leqq \sqrt{\frac{M}{t_{1}-\tau}},
$$

$$
\begin{aligned}
\iint_{\Omega_{t_{1}}}\left|K\left(x_{1}, t_{1} \mid \xi, \tau\right) K(\xi, \tau \mid x, t)\right| d t & \leqq M^{2} A \int_{t}^{t_{1}} \frac{d \tau}{\sqrt{t_{1}-\tau} \sqrt{\tau-t}} \\
& \leqq \pi M^{2} A,
\end{aligned}
$$

where $A$ is some constant. Hence that term remains finite. The $(n+2)$ th term is in absolute value not greater than the expression

$$
\frac{C\left\{D\left(t_{1}-t\right)\right\}^{n}}{n !}, \quad n \geqq 0,
$$

where $C$ and $D$ are certain constants, - provided of course that we are dealing with integrable functions. If we neglect for the moment this proviso, we see that the series, starting with the second term, is absolutely and uniformly convergent.

The first term is integrable over the given $x, t$ region for any given values 
of $x_{1}, t_{1}$, and is continuous in the neighborhood of any point $\left(x_{1}, t_{1} \mid x, t\right)$ provided that we do not have both $x_{1}=x$, and $t_{1}=t$. It is understood of course that $t_{1} \geqq t$. Let us now consider the remaining terms in regard to continuity, and incidentally therefore in regard to integrability.

The second term, as we have shown, remains finite, and is continuous except when we happen to have $x_{1}=x, t_{1}=t$. Let us then examine the value of this term in the four dimensional neighborhood of a point

$$
(X, T \mid X, T) \text {, }
$$

where $(X, T)$ is within $\Omega$, and show that by taking our neighborhood small enough, we can make the value of this term as small as we please $(\leqq \eta)$ provided that we keep $t_{1} \geqq t$; in other words, that the second term is continuous at any point $(X, T \mid X, T)$, if it is given there the value zero.

By referring to the explicit expression for $K\left(x_{1}, t_{1} \mid x, t\right)$, we see that the only term in $K_{2}\left(x_{1}, t_{1} \mid x, t\right)$ that requires examination is

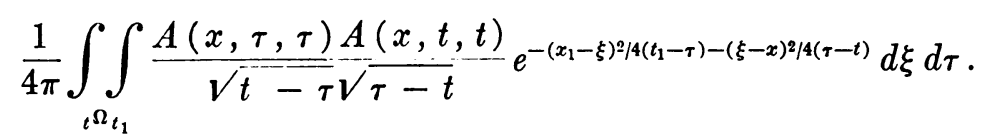

Let us divide the region of integration for $\xi$ into three parts, $c_{1}$ to $X-\Delta / 2$, $X-\Delta / 2$ to $X+\Delta / 2$, and $X+\Delta / 2$ to $c_{2}$, where $c_{1}$ and $c_{2}$ are respectively the minimum and maximum values of $x$ in $\Omega$. If we write $|A(x, t, t)|<N$, and make use of the inequality (14), we see that the above expression is in absolute value less than the expression

$$
\begin{aligned}
& \frac{N^{2} \Delta}{4}+\frac{N^{2}}{4 \pi} \int_{c_{1}}^{X-\Delta / 2} d \xi \int_{t}^{t_{1}} \frac{e^{-\left(x_{1}-\xi\right) / 4\left(t_{1}-\tau\right)-(\xi-x)^{2 /(t}(\tau-t)}}{\sqrt{t_{1}-\tau} \sqrt{\tau-t}} d \tau
\end{aligned}
$$

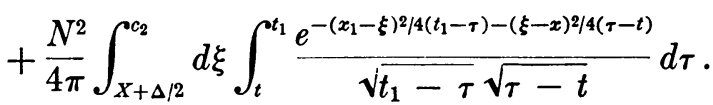

Let us now choose $\left|x_{1}-X\right|$ and $|x-X| \leqq \Delta / 4$, and $\Delta$ small enough so that the first term of the expression is less than $\eta / 3$. In the two integrals, the values of $\left|x_{1}-\xi\right|$ and $|\xi-x|$ will always be at least as great as $\Delta / 4$, and the integrands therefore less in absolute value than a certain constant, independent of the choice of $x$ and $x_{1}$ within the given interval. Hence if we take $\left|t_{1}-T\right|$ and $|t-T|<\epsilon$, keeping $t_{1} \geqq t$, we may choose $\epsilon$ small enough to make each integral separately in absolute value less than $\eta / 3$, and thus verify the desired inequality.

Accordingly the second term in (13) is continuous within the region. Hence, as is immediately verifiable, all later terms are continuous in the same region. If now we define $k\left(x_{1}, t_{1} \mid x, t\right)$ as the series (13), it will obviously satisfy 
the symmetrical relations (5). The continuous function $u(x, t)$ defined by (2) is now seen to be the same as the $u(x, t)$ determined by (10); for from the symmetry of the relations (5) and of the equations (2) and (10), it follows, in the usual way, that the $u(x, t)$ defined by (2) is the unique continuous solution of (10). Thus the closed form of the solution is established.

\section{The Generalized Differential and Integro-Differential Equations of Parabolic Type}

5. Introduction. The complication of the methods of $\$ \$ 1-4$, which are the classical methods, is due to the fact that we must insure the existence of $\partial^{2} / \partial x^{2}$ and $\partial / \partial t$ for functions which we wish to consider as possible solutions of any of the various parabolic equations. The possibility of avoiding these complications is suggested by trying to consider the complex of derivatives of highest order as a single differential operator

$$
\frac{\partial^{2}}{\partial x^{2}}-\frac{\partial}{\partial t}
$$

rather than as the combination of two.

Such a definition may be reached in several ways: we may for instance in forming this expression require that the increments of $x$ and $t$ continue to satisfy a certain relation as they approach zero.* It is more convenient for us, however, because closer to a possible physical interpretation and less laborious in its analysis, to define the operator by substituting for the parabolic differential equation

the generalized equation

$$
\frac{\partial u}{\partial t}-\frac{\partial^{2} u}{\partial x^{2}}=f(x, t)
$$

$$
\int_{\delta}\left[\frac{\partial u}{\partial x} d t+u d x\right]=\iint_{\sigma} f(x, t) d x d t
$$

in which $s$ is an arbitrary closed curve of a kind later to be described, and $\sigma$ is the enclosed region. $\dagger$ Similarly for the integro-differential equation

* We can generalize Green's theorem so that it applies to this operator. The corresponding operator has been defined by $\mathrm{H}$. Petrini in the elliptic case for Poisson's equation, Les dérivées premiers et secondes $d u$ potentiel, A c t a M a t e m a t i c a, vol. 31 (1908), p. 127-332, see introduction and page 181.

† Compare Professor Bôcher's treatment of the equation $\Delta^{2} u=0$ by means of the relation

$$
\int \partial u / \partial n=0 \text {. }
$$

M. Bôcher, On harmonic functions in two dimensions, P r oce edings of $\mathrm{th}$ e A me ric an A a demy of Science, vol. 41 (1905-06).

We may regard the passage from equation (1) to equation (2) as equivalent to defining

$$
\left(\frac{\partial}{\partial t}-\frac{\partial^{2}}{\partial x^{2}}\right)=\lim _{\sigma=0} \frac{1}{\sigma} \int_{s}\left[\frac{\partial}{\partial x} d t+() d x\right] \text {. }
$$

The corresponding definition is used by Ignatowsky, Die Vektoranalysis, Leipzig (1909-10), in his treatment of $\Delta^{2}$, but his treatment is not exact at all points. 


$$
\frac{\partial u}{\partial t}-\frac{\partial^{2} u}{\partial x^{2}}=\int_{T_{x}}^{t} A(x, t, \tau) u(x, \tau) d \tau
$$

we can substitute the generalized equation

$$
\int_{\delta}\left[\frac{\partial u}{\partial x} d t+u d x\right]=\iint_{\sigma} d \sigma \int_{T_{x}}^{t} A(x, t, \tau) u(x, \tau) d \tau .
$$

By establishing the corresponding generalization of Green's theorem we can give a treatment of the boundary value problems for these equations. It is not necessary that any derivative of $f(x, t)$ should exist, or that $f(x, t)$ should satisfy any restriction of the type of Levi's condition,*-a result that applies also to the corresponding generalization of the non-homogeneous elliptic equation. Likewise in the treatment of the integro-differential equation, it is unnecessary that any derivative of $A(x, t, \tau)$ should exist, or that $A(x, t, \tau)$ should satisfy Levi's condition.

6. Regular functions, standard curves, and Green's theorem. We say that a function is regular with reference to a generalized parabolic equation and a given region if with its first derivative with respect to $x$, it is continuous within and on the boundary of the region. The generalized parabolic equations are defined with reference to the integration of regular functions along certain classes of curves within the given region. These curves may be called standard curves and defined as follows.

A standard curve is a closed curve which does not cut itself at any point, composed of a finite number of branches. For each branch, the coördinates of a point are given by two functions $\phi(q)$ and $\psi(q)$, throughout a finite interval for $q ; \phi(q)$ and $\psi(q)$ are assumed to be continuous throughout the closed interval, and therefore finite, with their derivatives of the first order. It is assumed that $\phi^{\prime}(q)$ and $\psi^{\prime}(q)$ vanish only at a finite number of points, and do not both vanish at the same point. Hence no line parallel to the $x$-axis or $t$-axis can cut a standard curve in more than a finite number of points, unless it includes itself a branch of the curve.

We are now in a position to state Green's theorem:

If $u$ and $v$ are regular, and $f$ and $g$ are continuous, throughout a given simply connected region, and if for any standard curve $s$ contained wholly within the region

then

$$
\begin{array}{r}
\int_{\varepsilon}\left[\frac{\partial u}{\partial x} d t+u d x\right]=\iint_{\sigma} f d \sigma \\
\int_{\delta}\left[-\frac{\partial v}{\partial x} d t+v d x\right]=\iint_{\sigma} g d \sigma
\end{array}
$$

$$
\int_{\delta}\left[\left(v \frac{\partial u}{\partial x}-u \frac{\partial v}{\partial x}\right) d t+u v d x\right]=\iint_{\sigma}(v f+u g) d x d t .
$$

\footnotetext{
${ }^{*}$ Levi, loc. cit., § 8, Art. 23.
} 
7. Proof of Green's theorem. Let us form the functions

$$
\begin{array}{ll}
U=\int_{0}^{t^{\prime}} d t^{\prime} \int_{0}^{x^{\prime}} u\left(x+x^{\prime}, t+t^{\prime}\right) d x^{\prime}, & V=\int_{0}^{t^{\prime \prime}} d t^{\prime \prime} \int_{0}^{x^{\prime \prime}} v\left(x+x^{\prime \prime}, t+t^{\prime \prime}\right) d x^{\prime \prime} \\
F=\int_{0}^{t^{\prime}} d t^{\prime} \int_{0}^{x^{\prime}} f\left(x+x^{\prime}, t+t^{\prime}\right) d x^{\prime}, & G=\int_{0}^{t^{\prime \prime}} d t^{\prime \prime} \int_{0}^{x^{\prime \prime}} g\left(x+x^{\prime \prime}, t+t^{\prime \prime}\right) d x^{\prime \prime}
\end{array}
$$

keeping $\left|t^{\prime}\right|<a,\left|x^{\prime}\right|<a,\left|t^{\prime \prime}\right|<a,\left|x^{\prime \prime}\right|<a$, where $a$ is a positive constant less than the smallest distance of any point of $s$ to the boundary of the given region.

If we displace the standard curve $s$ parallel to itself by an amount whose components are $x^{\prime}, t^{\prime}$, it will still remain within the region, and equation (1) will give us, by the corresponding change of variable,

$$
\begin{aligned}
\int_{s}\left[\frac{\partial u\left(x+x^{\prime}, t+t^{\prime}\right)}{\partial x} d t+u\left(x+x^{\prime}, t+t^{\prime}\right)\right. & d x] \\
& =\iint_{\sigma} f\left(x+x^{\prime}, t+t^{\prime}\right) d x d t .
\end{aligned}
$$

If we integrate this equation from 0 to $x^{\prime}$ in regard to $x^{\prime}$ and from 0 to $t^{\prime}$ in regard to $t^{\prime}$ we shall have

(4) $\int_{s}\left[\frac{\partial U\left(x, x^{\prime} ; t, t^{\prime}\right)}{\partial x} d t+U\left(x, x^{\prime} ; t, t^{\prime}\right) d x\right]=\iint_{\sigma} F\left(x, x^{\prime} ; t^{\prime}, t^{\prime}\right) d x d t$,

since all the functions involved are continuous. But by a change of variable $\xi=x+x^{\prime}, \eta=t+t^{\prime}$, the expression for $U$ becomes

$$
U=\int^{t+t^{\prime}} d \eta \int_{x}^{x+x^{\prime}} u(\xi, \eta) d \xi,
$$

so that we have the formulæ

$$
\begin{aligned}
\frac{\partial U}{\partial x} & =\int_{t}^{t+t^{\prime}}\left\{u\left(x+x^{\prime}, \eta\right)-u(x, \eta)\right\} d \eta, \\
\frac{\partial U}{\partial t} & =\int_{x}^{x+x^{\prime}}\left\{u\left(\xi, t+t^{\prime}\right)-u(\xi, t)\right\} d \xi, \\
\frac{\partial^{2} U}{\partial x^{2}} & =\int_{t}^{t+t^{\prime}}\left\{\frac{\partial u\left(x+x^{\prime}, \eta\right)}{\partial x}-\frac{\partial u(x, \eta)}{\partial x}\right\} d \eta,
\end{aligned}
$$

all functions which are finite and continuous in $\sigma$ and on $s$. Hence

$$
\iint_{\sigma}\left(\frac{\partial U}{\partial t}-\frac{\partial^{2} U}{\partial x^{2}}\right) d \sigma=\int_{\sigma}\left[\frac{\partial U}{\partial x} d t+U d x\right] .
$$


But $F$ is continuous in $\sigma$, and therefore from (4) we have

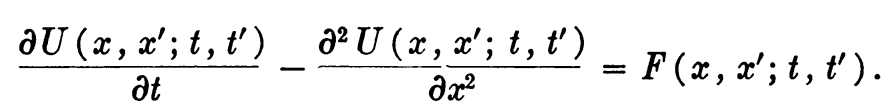

Similarly, we find the equation

$$
\frac{\partial V\left(x, x^{\prime \prime} ; t, t^{\prime \prime}\right)}{\partial t}+\frac{\partial^{2} V\left(x, x^{\prime \prime} ; t, t^{\prime \prime}\right)}{\partial x^{2}}=G\left(x, x^{\prime \prime} ; t, t^{\prime \prime}\right) \text {. }
$$

From these two equations it follows by means of Green's theorem, as ordinarily stated, that

$$
\begin{aligned}
& \int_{\sigma^{\prime}}\left[\left\{V\left(x, x^{\prime \prime} ; t, t^{\prime \prime}\right) \frac{\partial U\left(x, x^{\prime} ; t, t^{\prime}\right)}{\partial x}\right.\right. \\
& \left.\quad-U\left(x, x^{\prime} ; t, t^{\prime}\right) \frac{\partial V\left(x, x^{\prime \prime} ; t, t^{\prime \prime}\right)}{\partial x}\right\} d t+U\left(x, x^{\prime} ; t, t^{\prime}\right) \\
& \left.\quad \times V\left(x, x^{\prime \prime} ; t, t^{\prime \prime}\right) d x\right]=\iint_{\sigma}\left\{V\left(x, x^{\prime \prime} ; t, t^{\prime \prime}\right) F\left(x, x^{\prime} ; t, t^{\prime}\right)\right. \\
& \left.\quad+U\left(x, x^{\prime} ; t, t^{\prime}\right) G\left(x, x^{\prime \prime} ; t, t^{\prime \prime}\right)\right\} d \sigma .
\end{aligned}
$$

Referring to the definitions of $U$ and $V, F$ and $G$, we see that

$$
\begin{aligned}
\frac{\partial^{2} U}{\partial x^{\prime} \partial t^{\prime}} & =u\left(x+x^{\prime}, t+t^{\prime}\right), & \frac{\partial^{2} V}{\partial x^{\prime \prime} \partial t^{\prime \prime}} & =v\left(x+x^{\prime \prime}, t+t^{\prime \prime}\right), \\
\frac{\partial^{2} F}{\partial x^{\prime} \partial t^{\prime}} & =f\left(x+x^{\prime}, t+t^{\prime}\right), & \frac{\partial^{2} G}{\partial x^{\prime \prime} \partial t^{\prime \prime}} & =g\left(x+x^{\prime \prime}, t+t^{\prime \prime}\right) .
\end{aligned}
$$

Hence if we differentiate (5) in regard to $x^{\prime}, t^{\prime}, x^{\prime \prime}, t^{\prime \prime}$, under the integral sign, as we have a right to do, since the resulting functions are all finite and continuous, we get the formula

$$
\begin{aligned}
\int_{s}[\{v(x & \left.+x^{\prime \prime}, t+t^{\prime \prime}\right) \frac{\partial u\left(x+x^{\prime}, t+t^{\prime}\right)}{\partial x} \\
& \left.-u\left(x+x^{\prime}, t+t^{\prime}\right) \frac{\partial v\left(x+x^{\prime \prime}, t+t^{\prime \prime}\right)}{\partial x}\right\} d t+u\left(x+x^{\prime}, t+t^{\prime}\right) \\
& \left.\times v\left(x+x^{\prime \prime}, t+t^{\prime \prime}\right) d x\right]=\iint_{\sigma}\left\{v\left(x+x^{\prime \prime}, t+t^{\prime \prime}\right)\right. \\
& \left.\times f\left(x+x^{\prime}, t+t^{\prime}\right)+u\left(x+x^{\prime}, t+t^{\prime}\right) g\left(x+x^{\prime \prime}, t+t^{\prime \prime}\right)\right\} d \sigma .
\end{aligned}
$$

If in this we put $x^{\prime}=x^{\prime \prime}=t^{\prime}=t^{\prime \prime}=0$ we get equation (3), which we wished to prove.

If the boundary of the region is a standard curve the theorem will still hold if we 
take $s$ as the boundary itself. This can be shown by extending the function $u$ across $s$ in the following manner.

Let us isolate each vertex of the curve, and each point of the curve where there is a vertical tangent, unless it is an interior point of a vertical branch of the curve, by a small circle. Consider the portion of the curve between two successive circles. If this portion is vertical, we shall define $u$ and $\partial u / \partial x$ throughout a small neighborhood on the outside of the curve by means of the values which they possess on the vertical line itself for the same value of $x$.

If the portion is not vertical it will not have a vertical tangent at any interior point. Let us denote by $r(t)$ the values of $\partial u / \partial x$ along this portion of the curve, and define $u$ throughout a small neighborhood on the outside of the curve as that solution of the equation

$$
\frac{\partial u}{\partial x}=r(t)
$$

which takes on along the curve itself the already known values of $u$.

$I_{i} i$ now we surround each circle by a slightly larger circle, and take as a contour $s^{\prime}$ the curve composed, first, of the portions of $s$ between the successive circles, last constructed, and second of the portions of these circles interior to the region, we shall have for $s^{\prime}$, if we take the circles small enough, a standard curve, and it will lie wholly within a region for which $u$ and $\partial u / \partial x$ are continuous. Green's theorem will then hold for $s^{\prime}$. We get Green's theorem for $s$ if we let the circles approach zero as a limit.

8. The generalized parabolic differential equation. As the region to be considered, we shall understand that enclosed by the lines $t=t_{0}$ on the left, and $t=t_{1}$ on the right, and by the curves $x=\xi_{2}(t)$ above and $x=\xi_{1}(t)$ below. We shall assume that $\xi_{1}(t), \xi_{2}(t)$ and their first derivatives remain single-valued, finite, and continuous, and that $\xi_{2}(t)>\xi_{1}(t), t \geqq t_{0}$. The functions $\xi_{1}(t)$ and $\xi_{2}(t)$ are to have only a finite number of maxima and minima in the interval $t_{0} \leqq t \leqq t_{1}$.

Along the open boundary is to be given the continuous chain of values $(\alpha)$ of $\S 1$, under the conditions there stated. The function $f(x, t)$ is to be finite, and continuous within the given region.

Under these conditions there is one and only one regular solution $u(x, t)$ of the generalized parabolic differential equation $\left(1^{\prime}\right)$, which joins on continuously to the boundary values $(\alpha)$. It has the form

$$
u\left(x_{1}, t_{1}\right)=u^{\prime}\left(x_{1}, t_{1}\right)+\frac{1}{2 \sqrt{\pi}} \iint_{t_{0} \Omega_{t_{1}}} g\left(x, t \mid x_{1}, t_{1}\right) f(x, t) d x d t
$$

where $u^{\prime}\left(x_{1}, t_{1}\right)$ is the regular solution of the equation

$$
\frac{\partial u^{\prime}(x, t)}{\partial t}=\frac{\partial^{2} u^{\prime}(x, t)}{\partial x^{2}} .
$$


which joins on continuously to the boundary values $(\alpha),{ }^{*}$ and $g\left(x, t \mid x_{1}, t,\right)$ is the Green's function for this same equation.

From Green's theorem it follows that under the given conditions the solution of $\left(1^{\prime}\right)$ must necessarily have the form (6). On the other hand we can show directly that (6) does give us a solution which takes on the boundary values $(\alpha)$. In fact we shall show this if we show that the function

$$
z\left(x_{1}, t_{1}\right)=\stackrel{1}{2 \sqrt{\pi}} \iint_{t_{0} t_{t_{1}}} g\left(x, t \mid x_{1}, t_{1}\right) f(x, t) d x d t
$$

is regular with respect to $\left(1^{\prime}\right)$, takes on continuously the value zero on the open boundary, and satisfies $\left(1^{\prime}\right)$.

Let us write

and

$$
z(x, \tau)=Z(x, t)+w(x, t)
$$

$$
Z\left(x_{1}, t_{1}\right)=\frac{1}{2 \sqrt{\pi}} \iint_{t_{0} \Omega_{t_{1}}} \frac{1}{\sqrt{t_{1}-t}} e^{-\left(x_{1}-x\right)^{2 / 4\left(t-t_{1}\right)}} f(x, t) d x d t .
$$

We know that $Z\left(x_{1}, t_{1}\right)$ is regular with regard to $\left(1^{\prime}\right)$ since with its derivative in regard to $x$ it is continuous within and on the boundary of $\Omega . \dagger$ It is also a solution of $\left(1^{\prime}\right)$, as we can show by performing the indicated integration.

9. Formulæ of integration. Let $l$ be a straight line lying wholly within $\Omega$, of which the end points are $\left(a_{1}, b_{1}\right)$ and $\left(a_{2}, b_{2}\right)$, with $b_{2}>b_{1}$, and let $x^{\prime}$ be the $x$ coördinate of the point on that line whose other coördinate is $t$, i. e.

$$
x^{\prime}=\frac{a_{2}-a_{1}}{b_{2}-b_{1}}\left(t-b_{1}\right)+a_{1} .
$$

If we enclose this line in a small parallelogram, which we allow to vanish, we can easily deduce the following formulæ.

If $l$ is perpendicular to the $t$-axis, we have

$$
\begin{aligned}
\int_{a_{1}}^{a_{2}} d x_{1} \iint_{t_{0} \Omega_{t_{1}}} h_{01 / 2}\left(x, t \mid x_{1}, t_{1}\right) f(x, t) d x d t \\
=\lim _{\delta=0} \int_{t_{0} \Omega_{t_{1}-\delta}} d x d t f(x, t) \int_{a_{1}}^{a_{2}} h_{01 / 2}\left(x, t \mid x_{1}, t_{1}\right) d x_{1} .
\end{aligned}
$$

If $l$ is not perpendicular to the $t$-axis, we have

* For the existence and uniqueness of $u^{\prime}(x, t)$ under these conditions see G. E. Levi, loc. cit., $\S \S 2,5$.

† See E. E. Levi, loc. cit., p. 234 . See also $\$ 7$. 


$$
\begin{gathered}
\int_{a_{1} l_{a_{2}}} d x_{1} \iint_{t_{0} \Omega_{t_{1}}} h_{01 / 2}\left(x, t \mid x_{1}, t_{1}\right) f(x, t) d x d t \\
=\iint_{t_{0} \Omega_{b_{2}}} d x d t f(x, t) \int_{x^{\prime} l_{a_{2}}} h_{01 / 2}\left(x, t \mid x_{1}, t_{1}\right) d x_{1}, \\
\int_{b_{1} l_{b_{2}}} d t_{1} \iint_{t_{0} \Omega_{t_{1}}} h_{13 / 2}\left(x, t \mid x_{1}, t_{1}\right) f(x, t) d x d t \\
=\iint_{t_{0} \Omega_{b_{2}}} d x d t f(x, t) \int_{t^{l_{b_{2}}}} h_{13 / 2}\left(x, t \mid x_{1}, t_{1}\right) d t_{1} .
\end{gathered}
$$

In these formulæ, as before, we write

$$
h_{\alpha \beta}\left(x, t \mid x_{1}, t_{1}\right)=\frac{\left(x_{1}-x\right)^{a}}{\left(t_{1}-t\right)^{\beta}} e^{\left(x_{1}-x\right)^{2 / 4\left(t_{1}-t\right)},}
$$

from which it follows that

$$
\frac{\partial}{\partial x_{1}} h_{01 / 2}\left(x, t \mid x_{1}, t_{1}\right)=-\frac{1}{2} h_{13 / 2}\left(x, t \mid x_{1}, t_{1}\right) .
$$

10. Application to equation $\left(1^{\prime}\right)$. With reference to these formulæ let us form the expression

$$
\int_{C}\left[\frac{\partial Z}{\partial x_{1}} d t_{1}+Z d x_{1}\right],
$$

where $C$ denotes a closed polygon of a finite number of sides, the boundary of which does not anywhere cut itself. If we denote by ${ }_{t} C$ the portion of the contour composed of points of which the $t$-coördinates are greater than the value $t$, and by $b$ the maximum $t$-coördinate for any point of the contour, we shall have the equation

$$
\begin{aligned}
\int_{C} & {\left[\frac{\partial Z\left(x_{1}, t_{1}\right)}{\partial x_{1}} d t_{1}+Z\left(x_{1}, t_{1}\right) d x_{1}\right] } \\
\quad & =\frac{1}{2 \sqrt{\pi}} \iint_{t_{0} \Omega_{b}} d x d t f(x, t) \int_{t}\left[\frac{\partial h_{01}\left(x, t \mid x_{1}, t_{1}\right)}{\partial x_{1}} d t_{1}+h_{01 / 2}\left(x, t \mid x_{1}, t_{1}\right) d x_{1}\right] .
\end{aligned}
$$

The curvilinear integral of the second member of the above equation is however, as is well known, equal to

$$
\begin{array}{cl}
2 \sqrt{\pi} & \text { if the point }(x, t) \text { is inside } C, \\
0 & \text { if the point }(x, t) \text { is outside } C,
\end{array}
$$

since $h_{0 \frac{1}{2}}$ is itself a solution in $x_{1}, t_{1}$ of the homogeneous partial differential equation (7). The boundary points form an aggregate of zero external 
measure, and since the curvilinear integral remains finite, contribute nothing to the value of the second member. If then we divide $t_{0} \Omega_{b}$ into two parts, one consisting of the portion of $\Omega$ inside $C$, and the other the portion of $\Omega$ outside $C$, we observe that the value of the double integral over the latter portion is zero, on account of the zero factor in the integrand. Hence we have

$$
\int_{C}\left[\frac{\partial Z\left(x_{1}, t_{1}\right)}{\partial x_{1}} d t_{1}+Z\left(x_{1}, t_{1}\right) d x_{1}\right]=\iint_{C} f(x, t) d x d t,
$$

the double integral being extended over the interior of $C$.

Let us now finally consider any standard curve entirely within $\Omega$. In the first place it is rectifiable, and its length is by definition the limit of the perimeter of an inscribed polygon as the vertices of the polygon become infinite in number, in such a way as to make the polygon approach the contour. In order to construct the approximate polygons in a perfectly definite manner, we may imagine the $i$ th branch of the standard curve $s$ to have the coördinates of its points expressed in terms of the parameter $q$, as we have already described, and take as the vertices of the polygon the $n_{i}$ interior points of division corresponding to equally spaced values of $q$ in the interval (the vertices of the curve therefore are not vertices of the polygon). By taking every $n_{i}$ large enough, we get in this way a polygon of the kind already described, namely one of a finite number of sides which does not cut itself. If we denote the total approximate polygon by $C_{n}$, we shall have

$$
\int_{C_{n}}\left[\frac{\partial Z}{\partial x} d t+Z d x\right]=\iint_{C_{n}}^{0} f(x, t) d x d t .
$$

As each $n_{i}$ becomes infinite the right-hand member approaches the double integral over $\sigma$. The left-hand member approaches the integral over $s$, since the functions $Z$ and $\partial Z / \partial x$ are finite and continuous, and the curve $s$ is rectifiable. In fact the integral around $s$ may be regarded as the limit of a sum, the values of the functions being taken at the vertices of the polygons; and from the continuity of the functions $u$ and $\partial u / \partial x$ in the two-dimensional region it follows that the integral around $C_{n}$ can also be made to differ by as little as we please from that sum, and from its limit, by taking all the $n_{i}$ large enough.

The function $Z$ is therefore a solution of equation $\left(1^{\prime}\right)$.

Let $w(x, t)$ be that solution of (7) which has on the open boundary of $\Omega$ the same values as $Z(x, t)$, reversed in sign. Since $Z(x, t)$ is finite and continuous within and on the boundary of $\Omega, w(x, t)$ will be regular with respect to (7), as the existence theorem for (7) tells us. The function

$$
z(x, t)=Z(x, t)+w(x, t)
$$


will therefore be regular with respect to $\left(1^{\prime}\right)$, will take on zero values on the boundary of $\Omega$, and will satisfy $\left(1^{\prime}\right)$, for any standard curve lying wholly within the boundary. It follows moreover from Green's theorem that it must be expressible in the form (8). This is what was to be proved.

11. A generalized integro-differential equation. Let us consider now an integro-differential equation of parabolic type corresponding to the region $\Omega$. If we denote by $\int_{t_{0} t_{1}}$ integration along that portion of a line parallel to the axis of $t$ which is inside the region $\Omega$ and between the lines $t=t_{0}$ and $t=t_{1}$, the integral equation that we desire to consider may be written

$$
\int_{s}\left[\frac{\partial u}{\partial x} d t+u d x\right]=\iint_{\sigma} d x d t \int_{t_{0} t} A(x, t, \tau) u(x, \tau) d \tau,
$$

where by $s$ is meant any closed contour of the kind that we have already specified.

If $u(x, t)$ is continuous, the quantity

$$
\int_{t_{0} t} A(x, t, \tau) u(x, \tau) d \tau
$$

will be a continuous function of $x$ and $t .^{*}$ Hence by the theorem of $\S 8$, in order that $u(x, t)$ be the regular solution of (9), which takes on the boundary conditions $(\alpha)$ it is necessary and sufficient that it be a continuous solution of the equation

$$
\begin{aligned}
u\left(x_{1}, t_{1}\right)= & u^{\prime}\left(x_{1}, t_{1}\right) \\
& +\frac{1}{2 \sqrt{\pi}} \iint_{t_{0} \Omega_{t_{1}}} d x d t g\left(x, t \mid x_{1}, t_{1}\right) \int_{t_{0} t} A(x, t, \tau) u(x, \tau) d \tau .
\end{aligned}
$$

This equation, however, is merely an integral equation similar to that already discussed in part 1 . For by rewriting this equation in the form

$$
\begin{aligned}
& u\left(x_{1}, t_{1}\right)=u^{\prime}\left(x_{1}, t_{1}\right) \\
& \quad+\frac{1}{2 \sqrt{\pi}} \int_{c_{1}}^{c_{2}} d x \int_{t_{0} t_{1}} d t g\left(x, t \mid x_{1}, t_{1}\right) \int_{t_{0} t} A(x, t, \tau) u(x, \tau) d \tau,
\end{aligned}
$$

\footnotetext{
*According to our conditions the boundaries $x=\xi_{1}(t)$ and $x=\xi_{2}(t)$ cannot have portions consisting of horizontal straight lines. If it is desired to admit these, we must also generalize the theorem of $\$ 4$ to apply to functions $f(x, t)$ which are finite and discontinuous in a regular manner, - a generalization which is immediate.
} 
and interchanging the order of integration with respect to $t$ and $\tau$ we have the equation

$u\left(x_{1}, t_{1}\right)=u^{\prime}\left(x_{1}, t_{1}\right)$

$$
+\frac{1}{2 \sqrt{\pi}} \int_{c_{1}}^{c_{2}} d x \int_{t_{0} t_{1}} d \tau u(x, \tau) \int_{\tau t_{1}} g\left(x, t \mid x_{1}, t_{1}\right) A(x, t, \tau) d t,
$$

which is again the same as

$$
u\left(x_{1}, t_{1}\right)=u^{\prime}\left(x_{1}, t_{1}\right)
$$

$$
+\frac{1}{2 \sqrt{\pi}} \iint_{t_{0} \Omega t_{1}} d x d t u(x, t) \int_{t_{1}} g\left(x, \tau \mid x_{1}, t_{1}\right) A(x, \tau, t) d \tau .
$$

But this equation, according to the method of part 1 , has a unique continuous solution of the kind there specified. We have then the following

ThEоREм. There is one and only one regular solution $u(x, t)$ of the equation (9) which joins on continuously to the boundary values $(\alpha)$. It may be reduced to the form

$$
u\left(x_{1}, t_{1}\right)=u^{\prime}\left(x_{1}, t_{1}\right)-\iint_{t_{0} n_{t_{1}}}^{0} k\left(x_{1}, t_{1} \mid x, t\right) u^{\prime}(x, t) d x d t
$$

where $k\left(x_{1}, t_{1} \mid x, t\right)$ is the function associated to

$$
K\left(x_{1}, t_{1} \mid x, t\right)=\frac{1}{2 \sqrt{\pi}} \int_{t t_{1}} g\left(x, \tau \mid x_{1}, t_{1}\right) A(x, \tau, t) d \tau,
$$

by means of Volterra's relation.

\section{The Integro-Differential Equation of Hyperbouic Type}

12. In this last section we consider very briefly the hyperbolic integrodifferential equation of the following simple type,*

$$
\frac{\partial^{2} u(x, t)}{\partial x^{2}}-\frac{\partial^{2} u(x, t)}{\partial t^{2}}=\int_{0}^{t} A(x, t, \tau) \frac{\partial^{2} u(x, \tau)}{\partial \tau^{2}} d \tau,
$$

in which $A(x, t, \tau)$ is a continuous function, with continuous first derivatives, in a region

$$
\begin{gathered}
0 \leqq \tau \leqq t \leqq r \\
a-r+t \leqq x \leqq a+r-t .
\end{gathered}
$$

* This equation has been treated by the method of partial solutions by V. Volterra, Vibrazioni elastiche nel caso della eredita, $R$ e nd i c onti della $R$. A c c a d e mia dei $\mathrm{L}$ in e e i, vol. 21 (1912), p. 1. See also V. Volterra, Lectures delivered at the celebration of the 2Oth anniversary of the foundation of Clark University (1911), p. 80. 
The region of variation for the point $(x, t)$ is thus a triangle whose vertex is at the point $(a, r)$ and whose base is on the $x$-axis bounded by the points $(a-r, 0)$ and $(a+r, 0)$. The solution of this equation is to be regular, ${ }^{*}$ and is to satisfy the boundary conditions

$$
\begin{gathered}
u(x, 0)=f(x), \\
{\left[\frac{\partial u(x, t)}{\partial t}\right]_{t=0}=f_{1}(x),}
\end{gathered}
$$

in which $f(x), f_{1}(x)$ and their first derivatives are continuous functions of $x$ in the range of values $a-r \leqq x \leqq a+r$.

As long as we base our treatment on the characteristic function of Riemann, which is itself regular, there is no need of generalizing the equation in order to get rid of the second derivatives. We therefore limit ourselves in this section to a treatment that corresponds to $\S 1$, and content ourselves with a bare mention of the resulting theorem.

Theorem. Under the given conditions for $A(x, t, \tau)$ there is one and only one regular solution of $(1), u(x, t)$, which joins on continuously to the boundary values $(\beta)$. It may be reduced to the form

where

$$
u(x, t)=u^{\prime}(x, t)-\int_{x-t}^{x+t} d \xi \int_{0}^{\tau_{x}} l(x, t \mid \xi, \tau) u^{\prime}(\xi, \tau) d \tau,
$$

$$
\begin{aligned}
& \tau_{x}=t-x+\xi \text { for } \quad \xi \leqq x \\
& \tau_{x}=t+x-\xi \text { for } \quad \xi \geqq x,
\end{aligned}
$$

where $u^{\prime}(x, t)$ is the regular solution of the equation

$$
\begin{aligned}
& \frac{\partial^{2} u^{\prime}(x, \tau)}{\partial x^{2}}-\frac{\partial^{2} u^{\prime}(x, t)}{\partial t^{2}}-A(x, t, \tau) \frac{\partial u^{\prime}(x, t)}{\partial t} \\
& +\left[\frac{\partial A(x, t, \tau)}{\partial \tau}\right]_{\tau=t} u^{\prime}(x, \tau) \\
& \quad=-A(x, t, 0) f_{1}(x)+\left[\frac{\partial A(x, t, \tau)}{\partial \tau}\right]_{t=0} f(x)
\end{aligned}
$$

that takes on the boundary conditions $(\beta)$, and where $l(x, t \mid \xi, \tau)$ is the function associated to

$$
L\left(x_{1}, t_{1} \mid x, t\right)=\frac{1}{2} \int_{t}^{t_{x}} G\left(x_{1}, t_{1} \mid x, \tau\right) \frac{\partial^{2} A(x, t, \tau)}{\partial t^{2}} d \tau
$$

* That is, is to be continuous, with continuous first derivatives, and integrable second derivatives $\left(\partial^{2} / \partial x^{2}\right.$ and $\left.\partial^{2} / \partial t^{2}\right)$ in the given region $0 \leqq t \leqq r, a-r+t \leqq x \leqq a+r-t$.

Trans. Am. Math. Soc. 33 
by means of Volterra's relation. By $G\left(x_{1}, t_{1} \mid x, t\right)$ is denoted Riemann's characteristic function for equation (3).*

In the case of the region that we are considering Volterra's relation may be written as

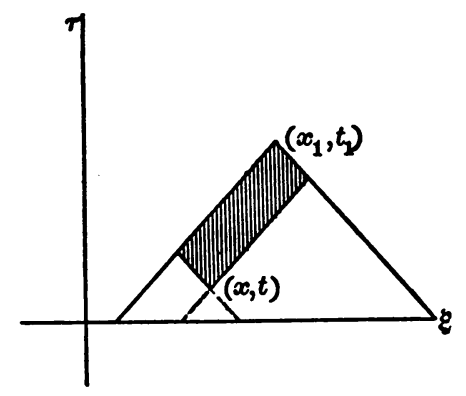

(5) $l\left(x_{1}, t_{1} \mid x, t\right)+L\left(x_{1}, t_{1} \mid x, t\right)=\iint L\left(x_{1}, t_{1} \mid \xi, \tau\right) l(\xi, \tau \mid x, t) d \xi d \tau$, the double integration being extended over the shaded area in the accompanying diagram.

The Rice Institute, June, 1914.

* That is, the solution of the equation adjoint to the first member of (3), which takes on the value 1 for $x=x_{1}, y=y_{1}$, and satisfies the conditions

and

$$
\sqrt{2} \frac{d G}{d s_{1}}=\frac{A(x, t, t)}{2} G \text { along the line } x-t=x_{1}-t_{1}
$$

$$
\sqrt{2} \frac{d G}{d s_{2}}=\frac{A(x, t, t)}{2} G \text { along the line } x+t=x_{1}+t_{1} \text {. }
$$

